\title{
Formación del Espíritu Empresarial en Jóvenes Mexicanos: Retos y Oportunidades en la Educación Media Superior
}

\section{Formation of the Entrepreneurial Spirit in Young Mexicans: Challenges and Opportunities in the Upper Secondary Education}

\author{
Joel Gilberto Olozagaste *1 \\ Yannet Paz Calderón ${ }^{2}$ \\ Mónica Teresa Espinosa ${ }^{2}$ \\ 1 Colegio Nacional de Educación Profesional Técnica (CONALEP) \\ 2 Universidad Tecnológica de la Mixteca, Oaxaca
}

\begin{abstract}
El Colegio Nacional de Educación Profesional Técnica (CONALEP), ubicado en Santiago Huajolotitlán, Oaxaca, México, se ha preocupado por el futuro de sus egresados y en concordancia con el Plan Nacional de Desarrollo 2007-2011, en 2008 incorporó el impulso al emprendimiento productivo, a través de la integración de la materia de Formación Empresarial, en los programas de estudio de su oferta educativa. En este trabajo se analizan las experiencias de emprendimiento de egresados del CONALEP, Plantel 145. Se hace énfasis en que estos emprendimientos se han desplegado bajo ciertas condiciones socioeconómicas y con determinados conocimientos adquiridos durante la formación técnica de los estudiantes, lo cual puede facilitar o no la creación de nuevas empresas por parte de ellos. La investigación es cualitativa, se realizaron entrevistas a egresados de esta institución para conocer la forma en que iniciaron y desarrollaron sus empresas. Los hallazgos indican que es necesario realizar diversas actividades académicas que refuercen el desarrollo de habilidades de emprendimiento para que los alumnos consideren que iniciar una empresa es una opción viable de autoempleo. Dados los índices de desempleo que prevalecen en el país es imperativo fomentar la creación de nuevas empresas, esta investigación contribuye a vislumbrar algunas alternativas encaminadas a impulsar y desarrollar el espíritu empresarial de egresados de instituciones de nivel medio superior como el Conalep.
\end{abstract}

Descriptores: México, Educación, Empresa, Joven.

The National College of Technical Occupational Education (CONALEP), located in Santiago Huajolotitlán, Oaxaca, Mexico, is concerned for the future of its graduates, and in accordance with the National Plan of Development 2007-2011, in 2008 it incorporated the impetus to productive entrepreneurship, through the integration of the subject of Business Training, in the study programs of its educational offer. This paper analyzes the Entrepreneurship experiences of CONALEP graduates, Campus 145. These ventures have been deployed under certain socio-economic conditions and with certain knowledge acquired during the technical training of students, which may or may not facilitate the creation of new enterprises by them. The research is qualitative, interviews were conducted with graduate students to know the way in which they started and developed their companies. The findings indicate that it is necessary to perform some academic activities that reinforce the development of entrepreneurship skills so that the students consider that starting a business is a viable option of self-employment. Given the unemployment rates prevailing in the country, it is imperative to encourage the creation of new companies, this research contributes to some alternatives aimed at promoting and developing the entrepreneurship of graduates from higher education institutions such as Conalep.

Keywords: Mexico, Education, Company, Youth.

*Contacto: joelgil_om@hotmail.com

ISSN: 0718-7378

www.rinace.net/rlei/
Recibido: 29/06/2017

$1^{\text {a }}$ Evaluación: 15/08/2017

Aceptado: 24/09/2017 


\section{Introducción}

En 2015 en México la población joven de 15 a 29 años fue de 30,6 millones, lo que representó el $25,75 \%$ de la población total. La tasa de desocupación en los jóvenes durante el primer trimestre de 2016 fue de $7,2 \%$, nivel superior a la tasa estimada a nivel nacional para la población de 15 y más años (que es de 4\%) (INEGI, 2016).

México no es el único país en el cual se ha notado un gran desinterés por parte de los jóvenes en términos de emprendimiento. En España, según Flash Eurobarometer Survey on Entrepreneurship, los niveles de personas que desean ser sus propios jefes han disminuido, es decir el $52 \%$ de las personas ha tomado el lado del empleo y dejado de lado esa mentalidad de empresario. Es necesario considerar las características de la población, ya que al tener culturas completamente diferentes tienden a enfrentar retos de mayor incertidumbre, como es el caso de Estados Unidos, en donde las personas tienen el $27 \%$ de inseguridad de negocio mientras que en España representa casi el 50\%, haciendo esto más complicado. Los jóvenes actualmente necesitan mayor motivación y competencia dentro de los sistemas educativos, pues la incorporación al mercado laboral se va haciendo cada vez más complicada, por temas en los rezagos educativos en los diferentes países, creando emprendedores con deficiencias y carentes de bases fuertes para competir.

Antes que nada, debemos afirmar que no existe un concepto definido de "emprender" o "emprendedor", ya que todos los autores que se lo han cuestionado, se basan en su observación y experiencia propia o ajena. Algunos autores consideran que emprender, más que una moda o una opción, es una necesidad, es el camino para que una economía se vitalice; es por ello que los emprendedores se vuelven el canal óptimo para el desarrollo y sostén de la economía y el desarrollo futuro de cualquier nación. La población más creativa e innovadora, capaz de generar valor a través de sus ideas y ponerlas en el mercado para satisfacer necesidades, además de crear empleos de alto valor es la de los jóvenes (Aguilar y Ocampo, 2014).

El emprendimiento productivo es importante, porque de acuerdo con algunos autores, es el eje principal en la creación de empresa, tal y como destaca Federico-Sabaté (2003): "Ayuda a construir o reconstruir circuitos de producción y circulación de bienes y servicios, enriqueciendo el entramado social, sirviendo de punto de incorporación de los sectores marginales y excluidos, ampliando el acceso al trabajo, captando y potenciando las capacidades y energías sociales existentes. (p. 16)

En México, a través del sistema educativo, se ha intentado fomentar el desarrollo de habilidades de emprendimiento en los estudiantes para que encabecen el surgimiento de nuevas empresas, legalmente constituidas, que contribuyan al desarrollo de la economía nacional, regional y local. Para el estado de Oaxaca la tasa de desempleo juvenil es de 7,7\% (STPS, 2015). Esto refleja un grave problema que enfrenta la población joven, por lo que "la creación de empresas es una vía de generación de riqueza y de empleos y, aún con escasas expectativas de rentabilidad y un horizonte incierto, existen personas que se inician en esta aventura” (Ibáñez, s. f., p. 17).

La idea central de este trabajo es explorar la experiencia de emprendimiento que han tenido los egresados del Colegio Nacional de Educación Profesional Técnica (Conalep), Plantel 145, General Antonio de León, ubicado en el municipio de Santiago Huajolotitlán, Oaxaca, México, poniendo énfasis en que estas ideas de negocios se han desplegado bajo ciertas condiciones socioeconómicas y con determinados conocimientos adquiridos durante su formación técnica, lo cual puede facilitar o dificultar la creación de nuevas 
empresas por parte de estos egresados. Esto contribuirá a vislumbrar algunas alternativas y propuestas para fortalecer el emprendimiento en instituciones como el Conalep.

El camino analítico propuesto en este trabajo, distingue cuatro etapas. En la primera se realizan algunas reflexiones teóricas en torno al concepto de emprendedor y de emprendimiento productivo. En la segunda se describe la metodología y contexto espacial del objeto de estudio. La tercera da cuenta de los principales hallazgos, a partir de las experiencias de emprendimiento de los jóvenes egresados del Conalep. En la última, se plantean algunas conclusiones.

\section{Marco teórico}

El tema del emprendimiento ha sido considerado como un aspecto clave para detonar el crecimiento económico en entornos latinoamericanos (Camacho, 2007), pero su importancia ha sido reconocida también por países europeos (Núñez y Núñez, 2016) y asiáticos (Karhunen et al., 2008). En tales investigaciones se reconoce que los jóvenes estudiantes de nivel medio superior y superior muestran, de manera natural, poco interés por emprender. Sin embargo, destacan el cambio que se genera en sus objetivos y planes de vida después de la capacitación, misma que, aunque con sus variantes, se implementó para permitirles desarrollar sus habilidades e identificar estrategias para enfrentar los obstáculos que perciben de inicio; además de vislumbrar beneficios tanto individuales como colectivos al convertirse en emprendedores.

Desde la edad media han existido diversas definiciones para el término emprendedor. Amaru (2008), destaca como un primer antecedente histórico de la definición, la del emprendedor y economista Richard Cantillon, que, en 1775, en su ensayo sobre la naturaleza del comercio en general, fue el primero en tratar el papel crucial del emprendedor en la economía. Este autor identificó al emprendedor como alguien que "asume riesgos al comprar servicios o componentes a un precio determinado con la intención de revenderlos más tarde a un precio indeterminado" (p. 3).

Hoselitz (1951) analiza las nuevas formas de empresas y describe a sus creadores como "personas arriesgadas, líderes, tomadores de riesgos, trabajadores superiores, innovadores, debido a que eran quienes invertían su dinero en una promesa de empresa" (p. 193). Ibáñez (s. f.) después de haber analizado la postura de diversos estudiosos del tema y tras haber realizado su propia investigación empírica, señala que no existe un acuerdo sobre el término; por lo cual concluye diciendo "ser emprendedor implica una manera de pensar, unas creencias, es un estado de la mente, un conjunto de comportamientos caracterizados por innovación, flexibilidad y creatividad" (p. 27). Esta postura es reforzada por Ibarra (2008), quien dice que un "emprendedor es una persona que sobresale entre los demás por tener una decisiva actitud de pasión” (p. 44).

Con respecto a las características que debe tener un emprendedor existen una corriente acerca de los emprendedores, que se enfoca más en sus aspectos personales y rasgos individuales. Por ejemplo, Drucker (1985) expresa que

El empresariado innovador ve el cambio como una norma saludable. No necesariamente lleva a cabo el cambio él mismo. Pero (y esto es lo que define al empresariado innovador) busca el cambio, responde a él y lo explota como una oportunidad. (p. 25) 
Ibarra (2008), señala las particularidades que debe tener un emprendedor, coincidiendo con varios autores y lo denomina un superhombre; con estos atributos construye el perfil del emprendedor cuyas características principales son: experiencia, personalidad, adaptabilidad, ciclo de vida, rol y preparación.

Licandro y Echeverriarza (2006), consideran que el término emprendimiento es un sinónimo de empresa (ya que se refiere a una unidad productiva); además envuelve también la idea de algo en proceso de creación. Por lo tanto, concluyen señalando que por lo general se habla de emprendimiento cuando una empresa está naciendo o cuando está llevando adelante un nuevo proyecto de negocios. En la mayoría de las regiones, la actividad económica es impulsada por toda clase de empresas motivadas por la fuerza local. Así, el crecimiento económico a corto o a largo plazo proviene principalmente de este emprendimiento de las nuevas iniciativas de las empresas locales.

Schumpeter (1950), cataloga al emprendedor como el individuo dueño de la idea del negocio, quien ejecuta esas ideas y las innova constantemente, lo que este autor denomina "destrucción creativa”; este emprendedor es el agente de cambios económicos, quien crea y desarrolla nuevas posibilidades desconocidas en el entorno económico, con tal importancia que es el núcleo de la innovación (Galindo y Méndez, 2008, p. 27).

En el mismo sentido, Pierre (2012) retoma la definición emitida en el 2003 por la OCDEOrganización para la Cooperación y el Desarrollo Económico donde se afirma que "el emprendimiento es una manera de ver las cosas y un proceso para crear y desarrollar actividades económicas con base en el riesgo, creatividad e innovación, al gestionar una organización nueva o existente" (p. 7). Y concluye diciendo que "el emprendimiento constituye fundamentalmente un proceso innovador, que excluye las imitaciones de empresas o las simples adquisiciones como aquellas realizadas por los gerentes" (p. 7).

En suma, y de acuerdo a los autores consultados, el emprendimiento productivo se define como el proceso de creación y desarrollo de nuevas empresas o el mejoramiento de algún proyecto productivo. Esta última definición fue la que se utilizó en esta investigación.

\section{Contexto espacial de estudio}

\subsection{El Colegio Nacional de Educación Profesional Técnica (Conalep)}

El Conalep ofrece carreras técnicas de 3 años (divididos en 6 semestres), los aspirantes pueden ingresar a partir de los 15 años, que es la edad promedio en la que terminan sus estudios de secundaria.Además de la preparación técnica, cursan el nivel medio superior (que es indispensable para ingresas al nivel superior, si así lo desean).

El Conalep desde sus inicios -está institución fue creada por decreto presidencial en 1978 como un Organismo Público Descentralizado del Gobierno Federal- se centró en responder a las necesidades de formación de cuadros técnicos que demandan las unidades económicas del aparato productivo del país, contribuyendo al desarrollo social de la población con un nivel de formación que permitiera a sus egresados una pronta inserción al mercado laboral, congruente con su área de especialización. Su oferta educativa se compone de 47 carreras agrupadas en 7 áreas de formación ocupacional, que se imparten a nivel nacional en 308 planteles, las cuales son: Planeación y Transformación; Mantenimiento e Instalación; Tecnología y Transporte; Salud; Electricidad y Electromecánica; Contaduría y Administración; y Turismo, (Conalep, 2010, 2016). Las 
carreras que conforman la oferta educativa se diseñan y actualizan de acuerdo con las necesidades del sector productivo en las diferentes regiones del territorio nacional, mediante mecanismos de vinculación con diferentes sectores de la sociedad. Las carreras técnicas que se ofrecen en cada plantel, se supone que están en función de las necesidades que el aparato productivo tenga en la región o municipios donde se encuentre ubicado el plantel.

De esta manera, en 2008 y derivado de su Plan Institucional 2007-20121 (que a su vez tuvo concordancia con el Plan Nacional de Desarrollo 2007-20122) incorporó a los programas de estudio la materia de Formación Empresarial para los alumnos de quinto semestre de todas las carreras, la finalidad de esta asignatura es "que el alumno elabore un proyecto de inversión aplicado a un pequeño negocio a través de la investigación de mercados para determinar la viabilidad del mismo" (SEP, 2014, p.11).

Si bien en el objetivo general del Conalep se siguió manteniendo la premisa de formar profesionales técnicos que se incorporen de manera inmediata al mercado laboral, con esta integración del concepto de emprendimiento a los planes de estudio se ampliaron las posibilidades que tiene el egresado del Conalep para sumarse al ámbito productivo del país. Es innegable que esta modificación obedece, como ya se mencionó antes, a la necesidad que el país tiene de creación de nuevas empresas que contribuyan a la generación de auto empleo y empleos. Así, los alumnos al egresar pueden incorporarse el mercado de trabajo, seguir con estudios de nivel superior o crear su propia empresa.

\subsection{Conalep, Plantel 145, Gral. Antonio de León}

El estado de Oaxaca está ubicado en la parte sur de la República Mexicana, en 2015 tenía 3,9 millones de habitantes, distribuidos en 570 municipios. En la entidad predomina la población joven, la edad media es de 26 años, la asistencia escolar de la población de 15 a 24 años es de 37,9\%, y el nivel promedio de escolaridad es de apenas 7,2 años. La edad en la cual los jóvenes dejan de acudir a la escuela es a partir de los 15 años y la inscripción en el sistema educativo de la población de 6 a 14 años es de 94,6\%, (INEGI, 2015).

Existen razones que explican esta tendencia de bajo nivel de asistencia escolar. Una de ellas se relaciona directamente con el hecho de que los jóvenes se ven obligados a trabajar para contribuir a solventar los gastos del hogar, es decir, la falta de recursos económicos obliga a que los jóvenes ingresen al mercado laboral. Esta inserción se da en situaciones de desventaja, como el bajo nivel de escolaridad y la falta de experiencia laboral, que dificulta el acceso a un empleo digno y bien remunerado.

En gran parte del estado la población joven contrae matrimonio a muy temprana edad; los hombres por lo general a los 16 años y las mujeres antes de cumplir los 20 años. Antes de los 18 años tienen su primer hijo; lo que constituye otro de los motivos de deserción en

\footnotetext{
${ }^{1}$ Una de las estrategias que se establece en este Plan Institucional del Conalep, relacionada con el emprendimiento es: "Estrategia 2.4. Formación emprendedora e incubación de empresas. Incubar empresas y desarrollar el espíritu emprendedor de la población estudiantil, aprovechando la infraestructura y capacidad técnica de los planteles para apoyar la fase de gestación y puesta en marcha de proyectos empresariales desarrollados por alumnos; así como fomentar un sentido crítico y reflexivo hacia la creatividad y la innovación en la población estudiantil” (SEP, 2008 p. 39).

${ }^{2}$ El Plan Nacional de Desarrollo 2007-2012 en materia educativa tuvo como objetivo principal que todas las acciones del sector educativo estuvieran encaminadas al fortalecimiento de una economía competitiva y generadora de empleos, poniendo énfasis en la importancia de la inversión en el desarrollo de capacidades y habilidades de la población. Se reconoce que en este periodo se estaba generando un aumento importante de la población en edad de trabajar, lo cual constituía un reto y una oportunidad para incorporar a esta nueva fuerza de trabajo a las diferentes actividades productivas del país (Conalep, 2016)
} 
los jóvenes de 15 a 19 años, ya que adquieren responsabilidades que los obligan a ingresar al mercado laboral en busca de una fuente de ingresos que les permitan enfrentar sus nuevas responsabilidades (Jiménez, 2015).

Oaxaca es una entidad con altos niveles de pobreza ${ }^{3}$; en el año 2014, el Consejo Nacional de Evaluación de la Política de Desarrollo Social (CONEVAL), indicó que el 66,8\% de la población se encontraba en situación de pobreza y el 23,8\% vivía en situación de pobreza extrema ${ }^{4}$. La tasa de desocupación estatal, de acuerdo con la Secretaría del Trabajo y Previsión Social (STPS), para el tercer trimestre del 2015, fue del 3,5\% y la tasa de informalidad laboral ascendió al 80,6\%.

El Conalep, Plantel 145, Gral. Antonio de León, se encuentra ubicado en el estado de Oaxaca, en la Región Mixteca ${ }^{5}$, en el municipio de Santiago Huajolotitlán, localizado a7 kilómetros del municipio de Huajuapan de León. De acuerdo con información proporcionada por las actuales autoridades educativas del plantel, el $49 \%$ de los alumnos son originarios de la Heroica Ciudad de Huajuapan de León, y el $51 \%$ proviene de las comunidades de ambos municipios. En su mayoría son egresados de telesecundarias ${ }^{6}$, lo cual es una desventaja, ya que los conocimientos científicos y tecnológicos con los que cuentan los egresados de dichas instituciones no son equivalentes a los adquiridos por los estudiantes de las secundarias urbanas que cuentan con un maestro para cada materia, lo que genera como consecuencia una frágil base de conocimientos que, en el largo plazo puede traducirse en menos oportunidades para los alumnos del Conalep. Además, en muchas ocasiones los egresados del Conalep tienen dificultades para comprender el lenguaje técnico empleado en las distintas carreras, ya que no hablan bien español porque pertenecen a algún grupo étnico (Conalep, 2015).

Una vez concluido el periodo de formación académica, la mayoría de los alumnos regresa a sus comunidades de origen. Dadas las condiciones económicas y de pobreza que prevalecen en ellas, los egresados del mencionado plantel, tendrán pocas posibilidades de encontrar fuentes de empleo que mejoren sus expectativas de vida?

Cabe señalar que este plantel se rige bajo las premisas y lineamientos definidos por el Conalep a nivel nacional. Por lo tanto, se adapta a los cambios y reformas diseñadas para el mejoramiento de la calidad en la educación. Debido a que en México hay muy poca

3 Una persona se encuentra en situación de pobreza cuando tiene al menos una carencia social (en cualquiera de los seis indicadores siguientes: rezago educativo, acceso a servicios de salud, acceso a la seguridad social, calidad y espacio de la vivienda, servicios básicos en la vivienda, y acceso a la alimentación) y su ingreso es insuficiente para adquirir los bienes y servicios que requiere para satisfacer sus necesidades alimentarias y no alimentarias (CONEVAL, 2014).

4. Una persona se encuentra en situación de pobreza extrema cuando tiene tres o más carencias sociales, de seis posibles, dentro del Índice de Privación Social y que, además, se encuentra por debajo de la línea de bienestar mínimo. Las personas en esta situación disponen de un ingreso tan bajo que, aun si lo dedicase por completo a la adquisición de alimentos, no podría adquirir los nutrientes necesarios para tener una vida sana (CONEVAL, 2014).

5 "Los mixtecos, el cuarto pueblo indígena más numeroso de México, después de los nahuas, los mayas y los zapotecos, se llaman a sí mismos en su idioma Ñuu Savi, lo que en español significa Pueblo de la lluvia. Los antepasados de los actuales mixtecos se asentaron en un vasto territorio que abarca el noroeste del estado de Oaxaca, el extremo sur del estado de Puebla y una franja en el oriente del estado de Guerrero. Los nahuas llamaron a esta región Mixtlan, Lugar de nubes, o Mixtecapan, País de los mixtecos. Desde la llegada de los españoles, en el siglo XVI, es conocida como la Mixteca. Con una superficie aproximada de 40,000 kilómetros cuadrados, la región Mixteca presenta un relieve abrupto y desigual y, por consiguiente, una variedad de microclimas y ecosistemas" (Mindek, 2003, p. 5)

6 "La telesecundaria es una modalidad educativa [...] que establece sus centros escolares en zonas de alta marginación social, con un maestro que atiende todas las asignaturas; que generalmente no cuenta con apoyos para el trabajo administrativo, responsabilidad que es asumida también por él, y que cuenta con recursos audiovisuales, informáticos e impresos como apoyo para las diferentes asignaturas", (SEP, 2010, p. 6).

${ }^{7}$ En enero de 2016, se realizó una entrevista con el director del plantel y la jefa de servicios escolares que proporcionaron dicha información. 
oportunidad de desarrollo de capacidades emprendedoras en niveles educativos previos a los estudios universitarios (SEP, 2014), esta institución desde el 2008 ha incluido en sus planes y programas de estudio, el impulso al emprendimiento productivo. Esta decisión obedece a lo que Kuratko (2005) señala como una explosión global de las escuelas que ofrecen carreras relacionadas con el área de administración o de negocios, en las cuales se han ofrecido programas especializados en emprendimiento, que además coinciden con las estrategias y políticas gubernamentales interesadas en el desarrollo de los emprendimientos productivos por parte de los jóvenes. También ha habido esfuerzos por implementar programas de emprendimiento en escuelas de ingeniería (Mohd, 2016) en las que se han hecho esfuerzos por lograr que, al culminar sus estudios, no se tenga únicamente egresados que busquen empleo, sino profesionistas que generen empleos.

También ha crecido el interés por evaluar las intenciones de emprendimiento, inclusive varios años antes de que éste se materialice (Mwiya et al., 2017). En cuanto al municipio de Santiago Huajolotitlán, la población hasta el 2015 fue de 4.083 individuos (SEDESOL, 2016a), de los cuales el 26\% se encuentra entre los 15 y 26 años. De acuerdo al Plan de Desarrollo Municipal 2011-2013 de Santiago Huajolotitlán, las principales actividades que se desarrollan en el municipio son: actividades agrícolas (cultivo de maíz, frijol, etc.), pecuniarias (pastoreo extensivo, etc.), comercio (tiendas, misceláneas, etc.) y trabajos diversos (peón de albañil y jornalero). Las familias recurren con mayor frecuencia al comercio, ya que es la actividad que genera mayores ingresos. La migración interna se da en familias que presentan bajos recursos económicos, siendo las Ciudades de México, Puebla y Oaxaca sus destinos más frecuentes para trabajar.

En el municipio de Huajuapan de León, hasta el año 2015, había un total de 77.547 habitantes, representando el 1,8\% de la población del estado, con una edad promedio de 24 años o menos; los jóvenes de entre 15 y 29 años representaron el $28.3 \%$ del total de la población del estado (SEDESOL, 2016b). En Huajuapan León predominan las empresas de tipo familiar, cuyas actividades centrales son los servicios y el comercio.

El nivel educativo promedio de la población de más de 15 años, en ambos municipios, es de 7,9 años, lo que indica que la población tiene habilidades para escribir, leer y hacer operaciones aritméticas básicas. En relación a la asistencia escolar, esta disminuye considerablemente a partir de los 15 años en estas dos comunidades (INEGI, 2015).

Este breve panorama muestra la complicada situación económica y la vulnerabilidad a la que están expuestos los jóvenes que viven en los municipios mencionados anteriormente. El Conalep, Plantel 145, Gral. Antonio de León, es una institución del nivel medio superior de nivel técnico, que se presenta como una opción para la población de bajos recursos económicos que aspira a una formación académica. Su oferta académica está integrada por las siguientes carreras: Profesional Técnico Bachiller (PTB) en Contaduría, PTB en Informática, PTB en Hospitalidad Turística, PTB en Salud Comunitaria.

\section{Metodología}

Esta investigación es de tipo cualitativo. Se buscó, a través de entrevistas y encuestas analizar la experiencia de emprendimiento de los egresados del Conalep, Plantel 145, Gral. Antonio de León, que iniciaron un negocio. En el plantel no se cuenta con una base de 
datos que dé seguimiento a egresados ${ }^{8}$, lo que representó una dificultad al momento de identificar a los sujetos de estudio. Esta investigación es de carácter exploratorio, se empleó una técnica no probabilística denominada bola de nieve ${ }^{9}$ para ubicar a los sujetos de estudio ${ }^{10}$ que cumplieran con las dos siguientes características: jóvenes egresados que hayan cursado la materia de Formación Empresarial y que hayan emprendido un negocio productivo.

En total se entrevistó y encuestó a nueve jóvenes, seis fueron ubicados en Huajuapan de León y tres en Santiago Huajolotitlán. Las entrevistas se efectuaron entre el 18 de abril y el 2 de mayo de 2016.

\section{Resultados}

\subsection{Condiciones generales del proceso de emprendimiento de los egresados}

$\mathrm{El}$ 44\% de los jóvenes que iniciaron un negocio manifestaron que fue a los 18 años cuando lo pusieron en funcionamiento y el resto lo hizo entre los 19 y 21 años de edad. Como puede verse, uno de cada dos jóvenes de estas comunidades marginadas que decidieron emprender, esperaron únicamente a cumplir la mayoría de edad y tomaron el reto de trabajar por su cuenta. En las entrevistas, estos jóvenes señalaron que veían muchas carencias en su comunidad y por ello decidieron armarse de valor y ser ellos quienes ofrecieran los productos o servicios que nadie más les había brindado.

La motivación que tuvieron para ello, fue que la mayoría deseaba ser independiente. El tipo de proyectos productivos que los egresados emprendieron, una vez concluida su formación profesional, está relacionado con el sector terciario de la economía (servicios y comercio): una casa de empeño, una papelería, dos autolavados y cinco empresas que brindan el servicio de café internet. Tal motivación se vio impulsada por un ejemplo que habían visto durante su infancia, ya que el $77 \%$ de estos jóvenes emprendedores cuenta con familiares que tienen un negocio, lo cual influyó en su decisión de emprender. Claramente puede verse la gran importancia del ejemplo que los jóvenes reciben del entorno familiar, ya que como pudo constatarse, no es frecuente que quienes no crecieron en un entorno empresarial, decidan emprender. Eso solamente ocurrió en el 23\% de los casos.

Cabe destacar que todos los emprendedores identificados son hombres y el $89 \%$ vive con su pareja, a consecuencia de la temprana edad a la que en estos municipios la población suele contraer matrimonio. Además, las mujeres aún se ven influenciadas por el rol de género que culturalmente les es impuesto, lo que constituye una explicación del por qué no emprenden. Estos resultados coinciden con la investigación realizada por Packham y sus colaboradores (2010), quienes llegaron a la conclusión de que la educación relacionada con temas de emprendimiento tiene una influencia positiva sobre las actitudes que impulsan la creación de nuevas empresas, sin embargo, sus resultados muestran

\footnotetext{
${ }^{8}$ Si bien a nivel federal desde 1999 se realiza un estudio de seguimiento de egresados, pero su finalidad fue conocer en qué condiciones se da la inserción laboral de los jóvenes del Conalep, estos estudios no consideran el emprendimiento productivo como parte de la incorporación de estos jóvenes al mercado de trabajo.

${ }^{9}$ Dicho procedimiento consiste en que al primer sujeto entrevistado se le pide apoyo para identificar a otros sujetos con características similares a él (Pénelas et al., 2012).

${ }^{10}$ Por lo que se hizo uso del contacto directo que se tiene con algunos egresados a quienes se les pregunto si conocían a algún exalumno que hubiese iniciado un negocio. Sumado a lo anterior, las redes sociales constituyeron un apoyo para ubicar a los jóvenes emprendedores del Plantel 145, Gral. Antonio de León.
} 
variaciones importantes dependiendo del país en el que se brinde la capacitación y del género de los estudiantes.

Por otra parte, del total de los jóvenes entrevistados que emprendieron un negocio productivo, ninguno manifestó haber tomado un curso o taller para emprendedores o microempresarios, simplemente lo hicieron en base a su experiencia laboral y a los conocimientos adquiridos en el Conalep. Esto muestra que resulta más importante fortalecer en los estudiantes las habilidades personales y la seguridad en ellos mismos, impulsando su capacidad de trazarse metas y tener ambición para alcanzarlas y no únicamente contar con conocimientos técnicos en alguna área.

El 55\% de ellos decidió arriesgarse estableciendo un negocio relacionado con el perfil de la carrera que estudiaron; tal es el caso del PTB en Informática, ya que durante su formación profesional desarrolla, de manera teórica y práctica competencias profesionales y habilidades necesarias para el mantenimiento y reparación de equipos de cómputo, instalación y desinstalación de programas de cómputo, instalación de redes, entre otros conocimientos.

A partir de la información se puede observar que quienes más emprenden son los egresados de Informática, seguidos de los del área de Contaduría y por último se encuentran los egresados de la carrera en Salud Comunitaria y Hospitalidad Turística, que prácticamente no tienen la inquietud de iniciar ningún tipo de negocios, probablemente porque les es más difícil ver la aplicación práctica de sus conocimientos, sobre todo en un negocio que implique una inversión modesta en la que sus padres podrían apoyarlos.

Durante las entrevistas, los egresados señalaron que el único acercamiento que tienen con el área de administración es en su último semestre, donde cursan la materia de Formación Empresarial, lo que consideran insuficiente para adquirir los conocimientos necesarios para desarrollar y poner en marcha una empresa.

El hecho de que los egresados de la carrera de PTB en Contaduría emprendan, puede explicarse porque su formación técnica está relacionada estrechamente con la actividad empresarial. Sin embargo, en el caso de los egresados de PTB en Informática, ellos son quienes más se atreven a emprender, lo que podría deberse a que tienen una sólida formación en conocimientos técnicos relacionados con el área de computación, ya que desde el primer semestre están en sus respectivos talleres para iniciar con las prácticas de su quehacer profesional y esto les da seguridad respecto a los conocimientos que adquieren.

Los hechos anteriores muestran que, aunque en una determinada formación de PTB no se incluyan materias de formación empresarial, si se apoya a los estudiantes para que lleven a cabo aplicaciones prácticas de los conocimientos adquiridos en el aula, ellos se sentirán más seguros de llevar a cabo estas prácticas por su cuenta, una vez concluidos sus estudios.

Otro aspecto que puede considerarse como un catalizador para el emprendimiento, tiene que ver con la seguridad personal y la experiencia que pueden adquirir los estudiantes, si se incorporan al ámbito laboral, aunque sea por periodos cortos. Esto pudo comprobarse con los resultados de la investigación, ya que el $78 \%$ de los jóvenes manifestó que antes de iniciar su negocio estuvo trabajando durante un tiempo, para adquirir competencias 
laborales, lo que contribuyo en parte, en la decisión de emprender ${ }^{11}$, además en algunos casos pudieron ahorrar dinero que después utilizaron para poner en marcha su empresa. También mencionaron que el apoyo económico que recibieron de sus padres fue transcendental.

El apoyo familiar, para todos los jóvenes entrevistados, fue importante durante todo el proceso de planeación y puesta en marcha del emprendimiento. Esta ayuda se materializó en diferentes formas: a) les prestaron el espacio físico para la instalación del negocio, b) algunos familiares como hermanos, madre o conyugue, los apoyan como empleados de mostrador, sin asignarles un sueldo fijo, sólo les otorgan una compensación o gratificación económica de manera esporádica o simplemente se sobreentiende que su pago se encuentra implícito en el beneficio que se tiene como familia, al poder contar con alimentación, vestido, casa y en su caso, apoyo para que sigan estudiando o para que reciban servicios de salud cuando se requiera. Se encontró que únicamente el $22 \%$ de estas empresas contaban con un empleado formal. Este resultado muestra que los emprendimientos analizados, por ser incipientes, se encuentran aún en una etapa de autoempleo y si logran su permanencia, será hasta años más tarde cuando podrán convertirse en fuentes de empleo para la comunidad en la que se encuentran ubicados.

Por otra parte, se pudo identificar que ningún negocio está legalmente constituido. Una de las justificaciones que los jóvenes señalaron es que carecen de los conocimientos necesarios para realizar los trámites para su formalización, además consideran que al ser empresas pequeñas no es necesario que estén formalmente constituidas. Esta situación no genera resultados positivos para la economía local, ya que a pesar de que se sigan creando empresas, al no encontrarse registradas formalmente, y no otorgar a los familiares que les apoyan todos los beneficios que establecen las leyes mexicanas para los trabajadores, sus empresas no tendrán más impacto, por lo que sólo contribuirán a incrementar la economía informal.

Con respecto a la sobrevivencia de estas empresas se encontró que el tiempo de funcionamiento ha estado directamente relacionado con la competencia que día con día ha ido creciendo, y con la falta de experiencia laboral y habilidades profesionales, solamente el $22 \%$ de estos empresarios no pudo desarrollar las estrategias que les permitieran enfrentar esta situación de forma eficiente, por tal motivo, tuvieron que cerrar sus negocios.

El periodo promedio de vida de los emprendimientos que han sobrevivido y que constituyen el $78 \%$, es de 2,5 años. Es importante indicar que dos empresas tienen cinco años de vida, cuatro tienen más de un año de funcionamiento y una lleva un año. Esto muestra que la preparación en términos de administración de empresas, les ha hecho falta, ya que la profesionalización de las empresas es muy importante para lograr su permanencia (Belasteguigoitia, 2012).

En el caso de las empresas que han sobrevivido, sus propietarios tomaron la decisión de utilizar dos estrategias fundamentales: a) diversificar el tipo de productos o servicios que ofrecían cuando inició la empresa y b) mejorar los productos y servicios que ofrecen al público, ambas estrategias les han permitido atraer más clientes. ${ }^{11}$ Esta experiencia laboral permitió ser disciplinados y organizados; también a interactuar con los clientes, proveedores y
aprender cómo se debe tratar a los empleados. 
La responsabilidad que estos jóvenes han adquirido al ser los dueños de sus empresas, ha representado un reto durante este periodo de ejercicio profesional; pero también ha habido ventajas, entre las que destacan:

- Autonomía e independencia económica de sus padres.

- Libertad en la toma de decisiones.

Aunque los jóvenes parecen tener como prioridad el logro de la independencia de sus padres, la realidad es que aunque ya hayan puesto una empresa, aún en esa etapa sus padres los siguen apoyando, por ejemplo, al dejar que ellos utilicen una parte de sus casas para el establecimiento del negocio y al permitirles que no paguen renta, lo que constituye un subsidio de la totalidad del alquiler y brinda a los jóvenes emprendedores la idea de que a pesar de encontrarse en una etapa inicial de la empresa, desde sus inicios empiezan a obtener ganancias. Otro apoyo que suelen brindarles es tramitándoles algún préstamo monetario, del cual, alguno de los padres queda como aval, lo que les facilita el camino.

La mayoría de estos jóvenes que emprendieron un negocio, ha contemplado la idea de ampliar las instalaciones del mismo, o abrir una sucursal. Aunque también analizan la posibilidad de iniciar otro proyecto productivo en otro sector de la economía. No obstante, la falta de recursos económicos ha sido el factor que ha detenido el inicio de la puesta en marcha de otros proyectos productivos.

\subsection{Conocimientos adquiridos en el Conalep}

Los jóvenes manifestaron que durante su estancia en el Conalep adquirieron los conocimientos que les permitieron desarrollar las habilidades y destrezas necesarias para ingresar al mercado laboral o continuar sus estudios a nivel superior, pero no fueron suficientes para emprender un proyecto productivo.

En el caso de los egresados de la carrera de PTB en Contaduría, los jóvenes indicaron que entre las asignaturas que fueron de utilidad para el comienzo de su emprendimiento productivo fueron: proceso administrativo, contabilidad básica, contabilidad de costos, control presupuestal y tesorería y normatividad para la constitución de las empresas, que les generaron una orientación en el proceso de creación y desarrollo de su negocio. Cabe destacar que los jóvenes de esta carrera técnica tienen una formación económicoadministrativa dada la naturaleza de su perfil técnico.

Los estudiantes de las carreras de PTB en Informática y en Salud Comunitaria, comentaron que al no contar con un perfil administrativo les resultó complicado el manejo de su negocio. No obstante, todos los jóvenes entrevistados coincidieron en que la materia de Formación Empresarial les generó una visión más amplia de cómo desarrollar y estructurar un proyecto productivo, ya que, al concluir el programa de estudios de esta asignatura, el estudiante puede ser capaz de estructurar un slogan, una misión, visión y un organigrama, entre otros aspectos relacionados con la gestión de un negocio. Para los estudiantes de PTB en Contaduría esta asignatura complementa su formación ya que les otorga los conocimientos relacionados con aspectos de emprendimiento productivo.

Sin embargo, para los alumnos de carreras distintas a la de PTB en Contaduría, la materia de Formación Empresarial, es el único acercamiento que tienen con el área económicoadministrativa, si bien esa materia les permite observar la actividad emprendedora como una opción laboral, no es suficiente para poder concretar una idea de negocio que abarque aspectos administrativos y financieros. 
Los jóvenes emprendedores entrevistados como parte de esta investigación iniciaron su proyecto bajo condiciones que reflejan la falta de fortalecimiento en aspectos de administración, trato al cliente, recursos humanos y desarrollo del negocio, lo que ha conducido a que estos negocios se desenvuelvan en un ambiente de alta fragilidad en diferentes aspectos relacionados con la administración y comercialización. Esto provoca que tengan pocas posibilidades de sobrevivir ante los embates de la competencia y de las características de le economía local, regional y nacional.

\section{Reflexiones finales}

La generación y desarrollo de empresas representa una vía a través de la cual se pueden generar empleos, aun dentro de un contexto de escasas expectativas de rentabilidad y un horizonte incierto. Existen personas que toman el riesgo de iniciar un negocio, representando una oportunidad para la generación de empleos y producción e impactando de manera positiva en la región.

La importancia de fomentar el emprendimiento productivo desde el aula representa una opción para el futuro en la vida de los jóvenes, ya que para emprender un proyecto productivo además de ser necesarias ciertas competencias profesionales, es indispensable tener habilidades que moldeadas a través de la educación pueden impulsar la creación de nuevas empresas y así contribuir con el desarrollo y crecimiento económico.

A pesar los esfuerzos realizados por el Conalep de integrar el emprendimiento a sus planes de estudio, hace falta reforzar esta área para que los egresados consideren como opción viable iniciar un negocio propio. Diversos autores (Amaru, 2008; Anzola, 2003; Bateman y Snell, 2009; Freire, 2011; Ibáñez, s. f.), coinciden en que todo emprendedor debe poseer habilidades como: autoconfianza, perseverancia, tolerancia al riesgo y a la incertidumbre, liderazgo, responsabilidad, compromiso y necesidad de logro. Se sugiere que las instituciones educativas realicen distintas actividades académicas que estén encaminadas en primer lugar, a despertar el interés en los jóvenes por tener un negocio propio y en segundo lugar, que busquen fortalecer las diferentes habilidades que debe tener un emprendedor. Todo ello debe ser realizado considerando el contexto socioeconómico y cultural en el cual viven los jóvenes estudiantes.

Las actividades académicas que podrían ponerse en marcha, una vez al año, en el Plantel 145 son:

- Un curso en la modalidad de taller que tenga como objetivo principal fomentar las habilidades de emprendimiento.

- Conferencias con empresarios de la región, considerando entre ellos a los egresados del Plantel 145, que tengan una empresa. Se propone que sean 4 conferencias al semestre, una cada mes.

- También se plantea la realización de viajes de estudio a empresas de diferentes giros, que puedan realizarse en un día.

- Se propone también la realización de un concurso de emprendimiento productivo, en el que los estudiantes deberán formar equipos para desarrollar una idea de negocios, que será expuesta ante un jurado que se encargará de elegir los tres mejores proyectos. 
Este trabajo de investigación es de utilidad para que las autoridades el Conalep consideren integrar dentro de sus planes y programas de estudio el impulso al desarrollo de habilidades de emprendimiento productivo, y que represente para los jóvenes una oportunidad de crear empresas y contribuir así con el desarrollo económico de su región. Esto debe hacerse teniendo siempre presente las particularidades que tiene la comunidad en la que se encuentra ubicado cada plantel en las diferentes regiones del país.

Esto debido a que las características o la dinámica de las actividades académicas destinadas a fortalecer el emprendimiento no pueden ser las mismas en todos los planteles del Conalep, cada lugar y región donde están ubicados son diferentes en muchos aspectos.

Es importante señalar que también es necesaria la participación activa de los diferentes niveles de gobierno, que pongan en funcionamiento programas de apoyo a emprendedores, pero que estén sustentados en diagnósticos que evalúen los recursos y necesidades de las comunidades donde se pretenda impulsar la actividad emprendedora, no solo de los jóvenes sino de la población en general.

Finalmente es necesario agregar que los programas gubernamentales de apoyo a emprendedores no deberían ser dádivas de dinero a fondo perdido que contribuyan muy poco a la creación y permanencia de emprendimientos productivos, sino opciones reales de emprendimiento que impulsen el crecimiento económico.

Esta investigación deja de manifiesto la necesidad de realizar más estudios relacionados con este tema.

\section{Referencias}

Aguilar, S. y Ocampo, A. (2014). De emprendedor a empresario, "haga que su negocio" ;sea negocio! Ciudad de México: Grupo Editorial Patria.

Amaru, A. (2008). Administración para emprendedores, fundamentos para la creación y gestión de nuevos negocios. Ciudad de México: Pearson.

Anzola, S. (2003). La actitud emprendedora. Ciudad de México: Mc-Graw-Hill.

Bateman, T. y Snell, S. (2009). Administración, liderazgo y colaboración en un mundo competitivo. Ciudad de México: Edamsa Impresiones S. A.

Belasteguigoitia, I. (2012). Empresas familiares: Dinámica, equilibrio y consolidación. Ciudad de México: McGrawHill.

Camacho, D. (2007). Hacia un modelo de emprendimiento universitario. Revista Apuntes del CENES, $27(43), 275-292$.

Conalep. (2010). Formando profesionales técnicos en México, Conalep, una apuesta al futuro. Ciudad de México: Vacha, S. A

Conalep. (2015). Programa de estudios del módulo de formación empresarial. Secretaria de educación pública. Ciudad de México: CONALEP.

Conalep. (2016). Historia del Conalep. Ciudad de México: CONALEP.

Drucker, P. (1985). La innovación y el empresario innovador. Barcelona: Ed. Edhasa.

Federico-Sabaté, A. (2003). Las empresas sociales ¿un componente sustancial para consolidar la economía social? En A. L. Abramovich (Coord.), Empresas sociales y economía social (pp. 3578). Buenos Aires: Universidad Nacional de General Sarmiento. 
Freire, A. (2011). Pasión por emprender, de la idea a la cruda realidad. Ciudad de México: Santillana.

Galindo M. y Méndez, M. (2008). Emprendedores y objetivos de política económica. Revista ICEInformación Comercial Española, 841, 29-40.

Hoselitz, B. F. (1951). The early history of entrepreneurial theory. Explorations in Economic History, 3(4), 193-213.

Ibáñez, M. A. (s.f.). Actitudes emprendedoras de los estudiantes universitarios. Medición y propuestas de actuación. Bilbao: Mensajero.

Ibarra, D. (2008). Los primeros pasos al mundo empresarial, una guía para emprendedores. Ciudad de México: Editorial Limusa.

INEGI. (2015). Encuesta intercensal 2015. Principales resultados Oaxaca. Ciudad de México: INEGI.

INEGI. (2016). Estadísticas a propósito del día internacional de la juventud (15 a 29 años) 12 de agosto. Recuperado de www.inegi.org.mx/saladeprensa/aproposito/2016/juventud2016_o.pdf

Jiménez, C. (15 de febrero de 2015). Oaxaqueños, 60 \% se casan o juntan a los 15 años. Grupo NVI noticias. Recuperado de http://www.noticiasnet.mx/portal/en/node/262932

Karhunen, P., Ledyaeva, S., Gustafsson-Pesonen, A., Mochnikova, E. y Vasilenko, D. (2008). Russian students' perceptions of entrepreneurship. Results of a survey in three St. Petersburg universities, entrepreneurship development e Project 2. Helsinki: HSE Mikkeli Business Campus Publications.

Kuratko, D. (2005). The emergence of entrepreneurship education: Development, trends, and challenges. Entrepreneurship Theory and Practice, 29(5), 577-597.

Licandro, O. y Echeverriarza, M. (2006). Reflexiones metodológicas para el diseño de proyectos productivos asistidos. Recuperado de http://www.unesco.org.uy/educacion/

Mindek, D. (2003). Mixtecos: Pueblos indígenas del México contemporáneo. Ciudad de México: CDI.

Mohd, A. (2016). Entrepreneurship education in an engineering curriculum. Procedia Economics and Finance, 35, 379-387.

Mwiya, B., Wang, Y., Shikaputo, C., Kaulungombe, B. y Kayekesi, M. (2017). Predicting the entrepreneurial intentions of university students: Applying the theory of planned behaviour in Zambia, Africa. Open Journal of Business and Management, 5, 592-610.

Núñez, L. y Núñez, M. (2016). Noción de emprendimiento para una formación escolar en competencia emprendedora. Revista Latina de Comunicación Social, 71, 1069-1089.

Packham, G., Jones, P., Miller, C., Pickernell, D. y Thomas, B. (2010). Attitudes towards entrepreneurship education: A comparative analysis. Education $\&$ Training, 52(9), 568-586.

Pénelas, A. Galera, C., Galán, M. y Valero, V. (2012). Marketing solidario. El marketing en las organizaciones no lucrativa. Madrid: Ediciones Pirámide.

Pierre, A. (2012). Una teoría sobre el emprendimiento regional en la economía del conocimiento. Ciudad de México: Pearson.

SEDESOL. (2016a). Informe anual sobre la situación de pobreza y rezago social I. Recuperado de http://diariooficial.gob.mx/SEDESOL/2017/Oaxaca_039.pdf

SEDESOL. (2016b). Informe anual sobre la situación de pobreza y rezago social II. Recuperado de http://diariooficial.gob.mx/SEDESOL/2017/Oaxaca_462.pdf

SEP. (2008). Programa institucional 2007-2012. Recuperado de www.conalep.edu.mx/ 
SEP. (2010). Modelo académico de calidad para la competitividad. Recuperado de http://www.conalepvallarta.edu.mx/modelo\%20academico.pdf

SEP. (2014). Transfieren modelo de emprendedores de educación media superior para fortalecer competencias en estudiantes. Recuperado de http://www.sems.gob.mx/es_mx/sems/

STPS. (2015). Información laboral. Recuperado de http://www.stps.gob.mx/gobmx/estadisticas/pdf/perfiles/perfil\%20oaxaca.pdf

\section{Breve CV de los autores}

\section{Joel Gilberto Olozagaste}

Estudiante de la Maestría en Administración de Negocios de la Universidad Tecnológica de la Mixteca. Licenciado en Ciencias Empresariales por la Universidad Tecnológica de la Mixteca. Profesor del Colegio Nacional de Educación Profesional Técnica (CONALEP), plantel 145, General Antonio de León. Líneas de investigación que cultiva: educación, empleo y desarrollo de emprendimientos productivos. ORCID ID: O00O-00030317-2353. Email: joelgil_om@hotmail.com

\section{Yannet Paz Calderón}

Doctora en Economía Política del Desarrollo por la Benemérita Universidad Autónoma de Puebla (2015). Profesora Investigadora adscrita al Instituto de Ciencias Sociales y Humanidades de la Universidad Tecnológica de la Mixteca, Oaxaca, México. Es miembro del CA-28 Administración, Cultura y Desarrollo Económico (CAACYDE) del Instituto de Ciencias Sociales y Humanidades. Líneas de investigación que cultiva: trabajo, empresa y desarrollo; y jóvenes, educación y mercado laboral. ORCID ID: O000-0001-5787-9763. Email: ypaz@mixteco.utm.mx

\section{Mónica Teresa Espinosa}

Doctora en Administración por la Universidad Autónoma de Querétaro (UAQ). Profesora Investigadora del Instituto de Ciencias Sociales y Humanidades, de la Universidad Tecnológica de la Mixteca, Oaxaca, México. Es miembro del CA-28 Administración, Cultura y Desarrollo Económico (CAACYDE) del Instituto de Ciencias Sociales y Humanidades. Líneas de investigación que cultiva: cultura organizacional, y perspectiva de género en las organizaciones. ORCID ID: OOOO-OOO3-3636-1755. Email: monitte2005@hotmail.com 